\title{
Small Marker Chromosome Identification in Metaphase and Interphase Using Centromeric Multiplex FISH (CM-FISH)
}

\author{
Octavian Henegariu, Patricia Bray-Ward, Sevilhan Artan, Gail H. Vance, \\ Mazin Qumsyieh, and David C. Ward
}

Department of Genetics (OH, PB-W, SA, MQ, DCW), Yale University School of Medicine, New Haven, Connecticut; and Department of Medical and Molecular Genetics (GHV), Indiana University School of Medicine, Indianapolis, Indiana

\begin{abstract}
SUMMARY: Multicolor karyotyping procedures, such as multiplex fluorescence in situ hybridization (M-FISH), spectral karyotyping, or color-changing karyotyping, can be used to detect chromosomal rearrangements and marker chromosomes in prenatal diagnosis, peripheral blood cultures, leukemia, and solid tumors, especially in cases where G-banding is not sufficient. A regular M-FISH analysis requires relatively large amounts of labeled DNA (microgram quantities), is not informative in interphase nuclei, hybridization can take up to 2 to 3 days, and unlabeled human chromosome-painting probes are not available commercially. Unique probes (plasmids, PAC), specific for centromeric or subtelomeric chromosomal regions, can replace the painting probes in M-FISH to address specific issues, such as the identification of marker chromosomes and aneuploidies. A set of plasmid probes carrying repetitive sequences specific for the $\alpha$-satellite region of all human chromosomes were combined in a metaphase assay and an interphase assay, allowing identification of aneuploidies in one hybridization step, on a single cytogenetic slide. The fluorophore-dUTP and the labeled antibodies required to label and detect the DNA probes can be prepared in any laboratory. All DNA probes can be easily isolated and labeled using common molecular cytogenetic procedures. Because of the repetitive nature of the probes, hybridization time is short, usually less than 1 hour, and the analysis can be performed with nonspecialized image-processing software. (Lab Invest 2001, 81:475-481).
\end{abstract}

S everal multicolor karyotyping procedures, such as $\circlearrowleft$ multiplex fluorescence in situ hybridization (MFISH), spectral karyotyping, or color-changing karyotyping (Henegariu et al, 1999; Schrock et al, 1996; Speicher et al, 1996) were introduced in the past several years and successfully used to identify complex structural and numerical chromosome aberrations. These techniques were applied in prenatal diagnosis (Uhrig et al, 1999), peripheral blood cultures, leukemia, and solid tumors (Haddad et al, 1998; Huang et al, 1998), especially in cases where G-banding was not sufficient to identify the chromosome of origin. Any multicolor karyotyping procedure requires relatively large amounts of labeled chromosome-painting probe DNA (microgram quantities), works only on metaphase chromosomes, takes up to 2 to 3 days to hybridize, and unlabeled chromosome-painting probes are not available commercially. As an alternative approach, the use of unique probes (plasmids, PAC), specific for centromeric or subtelomeric chromosomal regions, can replace the painting probes in M-FISH assays to ad-

Received November 9, 2000.

Address reprint requests to: Dr. Octavian Henegariu, Microarray Facility, MB\&B Departments, Yale University School of Medicine, WWW 5, 333 Cedar Street, New Haven, Connecticut 06510. E-mail: octavian.henegariu@yale.edu dress specific issues such as aneuploidy or marker chromosome detection. Probes specific for the $\alpha$-satellite region of human chromosomes have been successfully used for many years to identify major prenatal aneuploidies and numerical aberrations in human tumors (Huegel et al, 1995; Pergament, 2000; Ried et al, 1992; Zhao et al, 1998). In this article, we describe a M-FISH procedure combining the centromeric probes of all human chromosomes in a single assay. This approach allows the identification of marker chromosomes and other aneuploidies on a single cytogenetic slide in less than 2 hours. A similar procedure was proposed in abstract format by others (Heller et al, 2000; Nietzel et al, 1999) but was not detailed. Probes can be prepared, labeled, and combined in any cytogenetic laboratory, using standard molecular cytogenetic techniques, and the analysis can be performed on any computer platform using imaging software, such as Adobe Photoshop.

\section{Results and Discussion}

We used a set of $\alpha$-satellite probes combined in an algorithm, detailed in Figures 1 and 2, to achieve hybridization and separate detection of the centromeres of all human chromosomes (except 13 and 21) on the same slide. This novel M-FISH diagnostic procedure is called centromeric M-FISH (CM-FISH) and is used for one-step diagnosis of chromosomal 


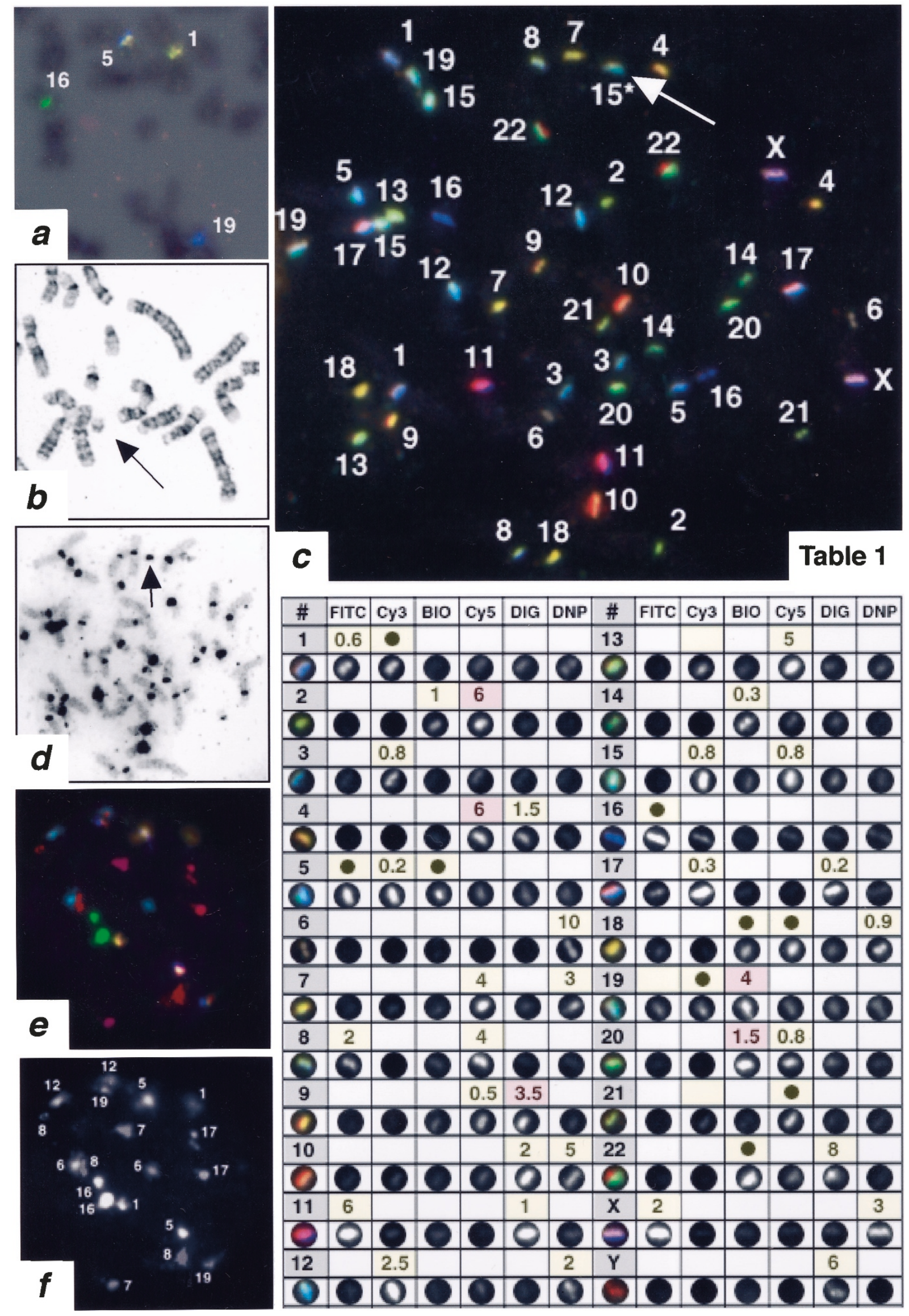


aneuploidies (Fig. 1). Two separate strategies were developed. In the first strategy, the metaphase assay or CM-FISH, human centromeric probes were labeled using combinatorial labeling, mixed together, and hybridized on the same cytogenetic preparation. This approach was used for small marker chromosome identification, and requires the presence of metaphases. In the second strategy, the interphase assay or interphase $\mathrm{CM}-\mathrm{FISH}$ (iCM-FISH), the centromeric probes were divided into three groups, which were hybridized on three separate areas of one cytogenetic preparation. This approach allows identification of aneuploidies in interphase nuclei, and does not require cell culture before FISH.

CM-FISH provides several advantages over other multicolor diagnostic procedures. The DNA probes do not require competitor DNA during hybridization, $\mathrm{FISH}$ signals from minute amounts of labeled DNA are strong, and all probes are available as plasmids (Choo et al, 1991; Durm et al, 1998), thus offering a virtually unlimited source of fresh DNA. Hybridization times of 0.5 to 2 hours are sufficient, allowing same-day diagnosis of a clinical sample. The main disadvantage is the lack of separation between the centromeres of chromosomes 13 and 21 (Maratou et al, 1999) and the inability to detect marker chromosomes lacking $\alpha$-satellite sequences (Magnani et al, 1998; Wandall et al, 1998).

\section{Metaphase Assay, CM-FISH}

This approach is especially useful in cases in which a small marker chromosome is diagnosed in a clinical sample by routine cytogenetic techniques (GTG banding). In most such cases, CM-FISH can identify the origin of the marker on the same day.

Combining centromeric probes in a CM-FISH assay created specific problems not encountered with M-FISH. Because of common alphoid subfamilies (Grady et al, 1992; Jorgensen, 1997; Lee et al, 1997; Mitchell, 1996; Sullivan et al, 1996; Wevrick and Willard, 1989), many centromeric DNA probes hybridize to several chromosomes simultaneously and yield hybridization signals with different sizes and strengths. To overcome this problem, the probes were labeled based on a six-fluorophore algorithm, including both combinatorial labeling (the same probe labeled with combinations of two or three fluorophores) (Ried et al, 1992) and ratio labeling (different amounts of dye to differentiate probes from one another) (Tanke et al, 1999). A combination of these labeling strategies allowed labeling of each DNA probe by no more than two fluorophores at the same time, thus facilitating separation of signals at the microscope (Fig. 1, Table). The nucleotides used were fluorescein isothiocyanate (FITC)-dUTP; Cy3-dUTP; Cy5-dUTP; digoxigenin (DIG)-dUTP, detected with anti-DIG-diethyl amino-

\footnotetext{
Figure 1.

a, Combinatorial and ratio labeling example: simultaneous use of three probes, pZ16a (fluorescein isothiocyanate, FITC), pC1.8 (Cy3), and pG-A16 (Cy5) allowed detection of four centromeres (chromosomes 1, 5, 16, and 19). Individual channel images (grayscale) for this hybridization are shown in Figure 2, a to C. b, Partial G-banded metaphase of a case with a small, unidentified marker chromosome (47, XX, mar). c, Centromeric multiplex fluorescence in situ hybridization (CM-FISH) analysis of a metaphase from the same case. The gray-scale images of the six channels (aminomethyl coumarin, AMCA; diethyl aminomethyl coumarin, DEAC; FITC; Cy3; Cy5; and Cy5.5) were combined and pseudocolored using PowerGene M-FISH software. Because various probes label chromosomes with different intensities, the imaging software merging and pseudocoloring the channels may display signals from unrelated chromosomes with similar color. Therefore, unlike regular M-FISH the marker was not identified by its color. Rather, after its position in the metaphase was identified (d), the marker was examined in all six channels and identified by its labeling pattern (Table 1). For one chromosome in every pair, Table 1 displays the hybridization signal in each of the six fluorescence channels separately. The 47th signal $\left(15^{\star}\right.$, arrow) in the metaphase (c) perfectly matches the pattern of hybridization corresponding to the two chromosomes 15 , indicating that the marker is of chromosome 15 origin. $d$, When using the fluorophore AMCA, DAPI counterstaining is not possible. However, overexposure of the same metaphase in the DEAC channel (2 to 4 seconds), clearly shows position of the small marker chromosome without requiring DAPI. e, Interphase CM-FISH analysis using mixture C1 on a bone marrow sample with trisomy 8. f, Black and white image of the same nucleus, with numbers indicating the position of every centromere. Individual channel images for this nucleus are depicted in Figure 2, d to i. Table 1, CM-FISH combination algorithm. The columns in the table depict the six fluorophores or haptenes used to label the probes. Numbers in the gray columns indicate the chromosome numeral or name. For each numeral, the corresponding row shows the number of microliters of the nick-translation-labeling reaction $(10 \mathrm{ng} / \mu$ l of DNA) used for one hybridization. These numbers show that some chromosomes required less probe than others to be detected. The occasional small black dots indicate that, for the respective channel, the centromere probe of a different chromosome will cross-hybridize there, and is part of the detection algorithm. For example, the black dot in the Cy3 channel for chromosome 1 indicates that this centromere will acquire a Cy3 hybridization from a different centromeric probe, in this case pC1.8. The colored CM-FISH image of every chromosome pair from image $\mathrm{c}$ is shown in the gray columns under the corresponding chromosome number. The row corresponding to every colored signal depicts the hybridization pattern in each of the six fluorescence channels. The light yellow cells indicate the channel(s) in which the respective chromosome was expected to show a hybridization signal. The signals can, but do not have to, be of similar strength for the analysis to work, because, for those chromosomes, ratio labeling is not important (for example, chromosome 12). An empty yellow cell indicates that the respective signal was sometimes absent, but did not affect chromosome identification. The light red cells indicate that ratio labeling was important for those chromosomes, and that the signal corresponding to the respective channel was the strongest. For example, chromosomes 4 and 9 both showed Cy5 and digoxigenin (DIG) hybridization signals, but Cy5 was stronger on chromosome 4, whereas DIG/DEAC was stronger on chromosome 9 . Because the metaphase shown in (c) did not have a $Y$ chromosome, the $Y$ hybridization pattern was added to the table from a male metaphase. Finally, because of the related alphoid families, many chromosomes had background hybridization in nonspecific channels. These signals were always weaker than the specific signals, were inconsistent (sometimes not present, depending on the slide quality and hybridization conditions) and did not interfere with chromosome identification Additional observations: Chromosome 1 acquired a Cy3 signal (black dot) from pC1.8. Chromosome 5 acquired a FITC signal from pZ16A and a biotin (BI0)/AMCA signal from pG-A16. Chromosome 16 acquired a FITC signal from pZ16A. Chromosome 19 acquired a Cy3 signal from pC1.8 and occasionally a FITC signal from pZ16A. Chromosomes 4 and 9, and 2, 18, and 20 were detected using ratio labeling. This was necessary as the centromeric probes of chromosomes 4 and 9 , and of 2, 18, and 20, respectively, cross-hybridize to one another to some extent. The cross-hybridization of L1.84 to chromosomes 2 and 20 is not very apparent (no dinitrophenyl [DNP] or Cy5.5 signals). However, probes from chromosomes 2 and 20 (pBS4D and pZ20) hybridized to the chromosome 18 centromere, thus the expected Cy5 and BIO/DEAC signals (black dots). Because of this, centromere 18 will have a different signature than 2 or 20 simply by adding the DNP-labeled L1.84 Thus, whereas centromeres 2 and 20 were identified by true ratio labeling, centromere 18 had a characteristic signature. Chromosomes 13 and 21 were detected with the Cy5-labeled probe L1.26 and both occasionally presented a faint Cy3 signal, probably from the cross-hybridization of probe p3-9. Chromosomes 14 and 22 were both detected by the BIO-labeled $\alpha$-XT(680). Their separate identification was achieved using the DIG/DEAC labeled p22/1:2.1;2.8;0.73, which did not visibly cross-hybridize with chromosome 14 .
} 

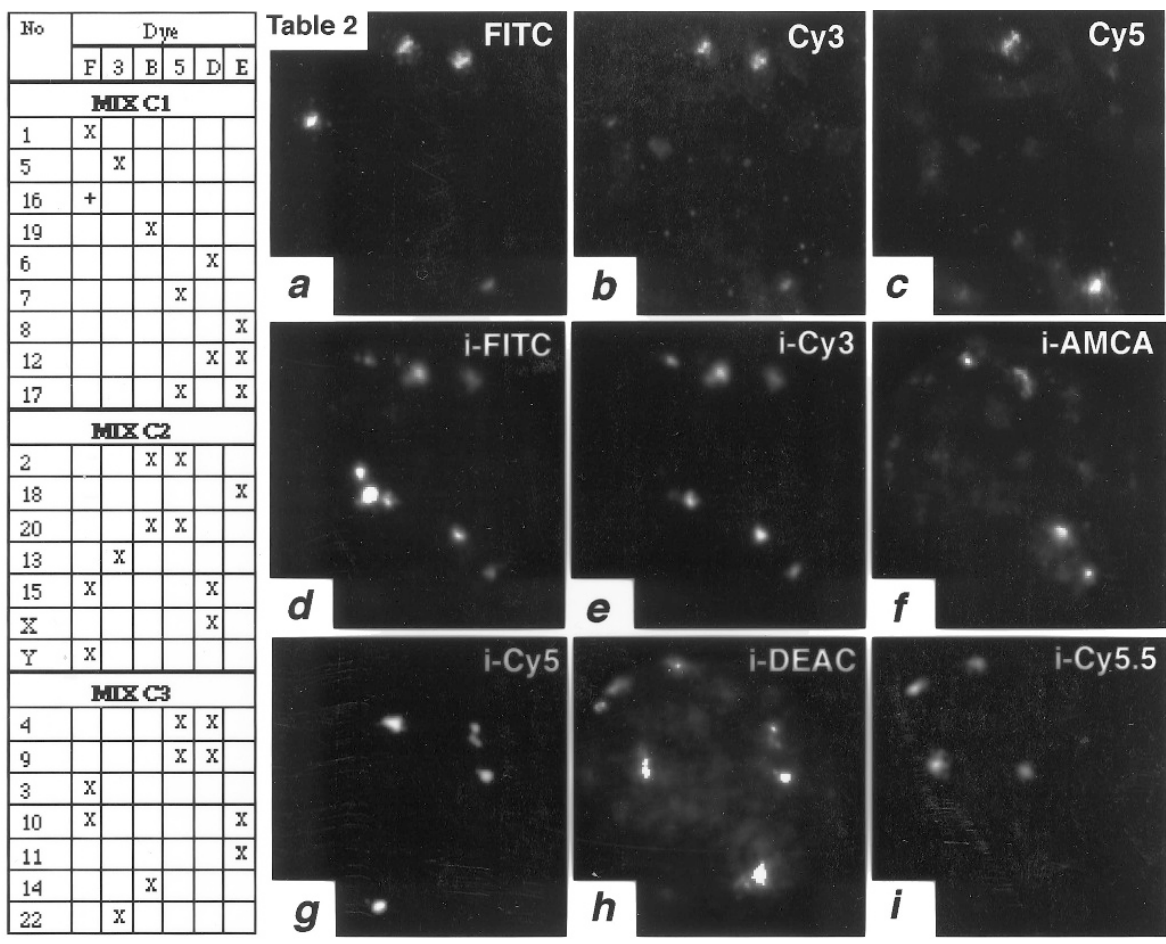

Figure 2.

Table 2: Interphase CM-FISH algorithm. Mixtures C1, C2, and C3 can identify aneuploidies in interphase nuclei (the probe for chromosome 13 in mix C2 identified both chromosomes 13 and 21). $x$ indicates the fluorophore(s) used to identify the various chromosomes. Detection of centromeres with shared alphoid families (group A centromeres) is achieved by combinatorial plus ratio labeling. $F=$ FITC; $3=$ Cy3; $B=\mathrm{BIO}$ (AMCA or Cy7); $5=\mathrm{Cy} 5 ; D=\mathrm{DIG} / \mathrm{Cy} 5.5$; and $E=\mathrm{DEAC}$. a to $\mathrm{c}$, Probe pZ16a (labeled with FITC) hybridized to the centromeres of 1, 5, 16, and very weakly to 19 (a); probe pC1.8 (labeled with Cy3) hybridized to 1, 5, weakly to 19 , and very weakly to 16 (b); pG-A16 (labeled with biotin, detected with avidin Cy5) hybridized to 5 and 19 (c). Simultaneous use of these three probes allowed identification of all four centromeres (see Fig. 1a), based on color combinations plus color ratios. Thus, the chromosome 1 centromere will be labeled with FITC and Cy3; the chromosome 5 centromere with FITC, CY3, and Cy5; the chromosome 16 centromere with FITC and weakly with Cy3; and the chromosome 19 centromere with Cy5, Cy3, and weakly with FITC. $d$ to i, As part of the C1 mixture, the same three probes plus probes for other four centromeres were used in an interphase CM-FISH analysis (Fig. 1, e to f) to detect a trisomy 8 in a tumor sample.

methyl coumarin (DEAC); dinitrophenyl (DNP)-dUTP, detected with antiDNP-Cy5.5; and biotin (BIO)-dUTP, detected with avidin Cy7 or aminomethyl coumarin (AMCA). Cy3.5 and Texas Red (fluorophores with similar absorption/emission characteristics) could not be used in the assay, because a strong Сy 3 signal was detectable through the Cy3.5 filter. Simultaneous use of all centromeric probes required a low posthybridization washing stringency $(0.2 \times$ SSC, $15 \mathrm{~min}$ utes at $42^{\circ} \mathrm{C}$ ). Although complex, this multiplex approach allowed simultaneous detection of all centromeres. To decrease cross-hybridization, the procedure could benefit from replacing some of the plasmid probes with labeled oligonucleotide probes (Warburton et al, 1991) with higher specificities for the respective chromosomes.

Based on their hybridization characteristics, the DNA probes were divided into two arbitrary groups. Group A included probes hybridizing to chromosomes with shared alphoid subfamilies (chromosomes 1, 5, 16, and 19;2, 18, and 20; 4 and 9; 13 and 21; and 14 and 22). Group B included probes hybridizing primarily to only one chromosome. Group A probes required combinatorial and ratio labeling, whereas group B probes required combinatorial labeling only (Fig. 1, Table and Fig. 2). To illustrate this, the DNA probes for chromosomes 4 and 9 , which partially cross-hybridize to one another, were both labeled with Cy5 and DIG (combinatorial labeling). The Cy 5 signal was stronger on chromosome 4, and the DIG/DEAC signal was stronger on chromosome 9, this difference allowing chromosome identification (ratio labeling). A particular case is that of chromosomes 13 and 21, which cannot be separately identified by FISH with $\alpha$-satellite probes. If a marker for 13 or 21 is found, it can be subsequently identified using painting probes or pericentromeric unique probes, such as PAC (B39l12 and 126N20), on chromosome 21 and YAC (748f2 and 967b1) on 13. In over 15 cases tested by CM-FISH (both normal controls and clinical samples), the hybridization patterns on every chromosome pair were constant. The only chromosomes showing FISH signals of variable intensities even by visual inspection, were chromosomes 13 and 21.

$\mathrm{CM}-\mathrm{FISH}$ does not require karyotyping software, as it is performed on cases already known cytogenetically by G-banding. In CM-FISH, once the marker is located in the metaphase, either by $4^{\prime}, 6$-diamidino-2phenylindole (4') (DAPI) counterstaining or by overexposing the image in the DEAC or FITC channel (where tissue auto-fluorescence is highest), only the fluorescence signals on the marker are visualized or captured in all six channels. The marker is identified by interpreting the hybridization pattern. Image analysis and 
pseudocoloring can be easily performed with generic image processing software, such as Adobe Photoshop.

\section{Interphase Assay, iCM-FISH}

The iCM-FISH approach allows detection of all centromeres in interphase nuclei on the same slide, an important advantage for early diagnosis of aneuploidies in leukemia, solid tumors, and in prenatal screening. Three separate probe combinations (mixtures C1, C2, and C3) (Fig. 2, Table) were necessary to reliably detect all centromeres, and six fluorophores were required to label all DNA probes. The three probe mixtures were simultaneously hybridized on different areas of the same slide. Efforts were made to keep all probes used for a particular type of analysis (such as prenatal diagnosis) in the same mixture. However, this was not always possible, because attention was also paid to preventing each probe mixture from becoming too complex. In every nucleus, any one of the six fluorophores used showed four to six hybridization signals. Of the three mixtures created (Fig. 2), C1 was aimed more at diagnosing aneuploidies in leukemia, whereas C2 was aimed more at prenatal diagnosis. Mix C1 was the most complex, as it included probes yielding six fluorescent signals in three channels (Fig. 2). Interphase CM-FISH requires a good slide preparation technique (Henegariu et al, 2001), to make the nuclei as "flat" (bi-dimensional) as possible. To test the procedure, we used bone marrow and peripheral blood samples, in which virtually all nuclei showed the specific aneuploidy. In this study, approximately $3 \%$ (bone marrow) and 8\% (peripheral blood) of all nuclei showed analyzable signals in all six fluorescent channels. The low numbers are explained by the complexity of the probe mixture: in each channel, four or six centromeres must have been simultaneously visible and the signals must have been in the same plane of the image. Additionally, some centromeres work better than others, and not all labeling reactions are identical. Small variations in labeling and sample preparation have a much higher impact in signal quality when using these probes than when using chromosome-painting libraries. The numbers found are in accordance with theoretical calculations: when counting centromere signals in each channel (one fluorophore), we found that approximately $60 \%$ of the nuclei had all expected 4 to 6 centromeric signals visible in the same plane. If the hybridization efficiency is identical for each of the six fluorophores used in an assay, $60 \%$ useful nuclei/channel should result in $4.7 \%$ of nuclei having separable signals in all channels, a number close to the experimental values. In general, the number of informative nuclei is higher for peripheral blood cultures because of the better nuclear morphology after spreading. Once an aneuploidy is identified by iCM-FISH screening, to count the number of interphase signals of that probe in a few hundred nuclei, the more convenient approach is to hybridize a separate slide with the respective centromeric probe labeled with a visible fluorophore (such as
FITC, Cy3, or rhodamine). This is useful especially when the respective aneuploidy is identified by an iCM-FISH probe, which happened to be labeled by an infrared dye (Cy5, Cy5.5, or Cy7). All infrared dyes require a CCD camera for visualization. Separate hybridization with a centromeric probe is easy, short (30 minutes), and the signals are easy to score in numerous nuclei. It is conceivable that some researchers would wish to hybridize all centromere probes on one slide and have the flexibility of scoring hybridization signals in hundreds of nuclei on the same slide. This would require the use of only visible fluorophores for labeling. Consequently, the number of probes in a set would need to be reduced. A simple solution is to divide each of the three centromere sets described (C1, C2, and C3) into two subsets and hybridize each subset on a different area of the same cytogenetic slide. In our laboratory, up to eight different hybridizations could be simultaneously performed on the same slide, by cutting $22 \times 22 \mathrm{~mm}$ coverslips into quarters, using a regular diamond pen. Eight such coverslip pieces can easily fit on one slide. For laboratories equipped with more sophisticated equipment and commercial software packages (such as the PowerGene M-FISH package), scoring infrared signals in many nuclei is possible by using the live camera view provided by the software. This enables visualization and counting of the infrared dye signals on the computer screen.

The main advantage of iCM-FISH is the use of interphase nuclei, with no need for time-consuming cell culture. All $\alpha$-satellite sequences can be identified within 1 to 2 hours from the start of the analysis. As with regular $\mathrm{CM}-\mathrm{FISH}$, the main disadvantage of the procedure is the lack of separation between chromosomes 13 and 21, a problem which can be addressed by using pericentromeric bacterial artificial chromosome, PAC, or yeast artificial chromosome clones on either one of these two chromosomes.

\section{Materials and Methods}

\section{DNA Probes}

The following centromeric probes $(\alpha)$ were used: $\alpha 1=$ pZ16A* (from H.F. Willard, Department of Genetics and Center for Human Genetics at Case Western Reserve University and the Research Institute of University Hospitals of Cleveland, Ohio, similar to pE25b from the American Type Culture Collection, ATCC, Rockville, Maryland); $\alpha 2=$ pBS4D* (from A. Baldini, Department of Pediatrics, Baylor College of Medicine, Houston, Texas); $\alpha 3$ = p3-9 (from H.F. Willard); $\alpha 4=$ pG-Xball/340* (from T. Hulsebos, Department of Human Genetics, Academic Medical Center, University of Amsterdam, The Netherlands); $\alpha 5=\mathrm{pC} 1.8$ (from T. Hulsebos); $\alpha 6=\alpha \mathrm{RI} 12$ (from ATCC); $\alpha 7=\mathrm{pZ7B}$ (from H.F. Willard); $\alpha 8=$ pJM128 (from ATCC); $\alpha 9=$

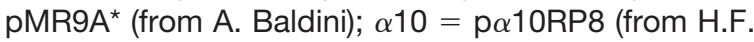
Willard); $\alpha 11=$ pLC11A (from H.F. Willard); $\alpha 12=$ p $\alpha 12$ H8 (from ATCC); $\alpha 13 / 21=$ L1.26* (from P. Devilee, Department of Human and Clinical Genetics, 
Leiden University Medical Center, The Netherlands); $\alpha 14 / 22=\alpha \mathrm{XT}(680)^{\star}$ (from A.L. Jorgensen, Department of Molecular and Structural Biology, University of Aarhus, Denmark); $\alpha 15$ = pTRA-25 (from A. Choo, The Murdoch Children's Research Institute, Royal Children's Hospital, Melbourne, Australia); $\alpha 16=$ see $\alpha 1$ (pSE16 can be added to the CM-FISH); $\alpha 17=$ p17H8 (from H.F. Willard) or pYAM 7-29 (from Y.B. Yurov, National Research Centre of Mental Health, Russian Academy of Medical Sciences, Moscow, Russia); $\alpha 18$

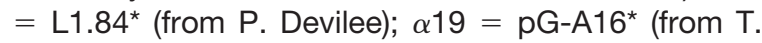
Hulsebos); $\alpha 20=$ pZ20* (from A. Baldini); $\alpha 21=$ see 13; $\alpha 22=$ p22/1:2.1;2.8;0.73 (from H.E. McDermid, Department of Biological Sciences, University of Alberta, Edmonton, Alberta, Canada); $\alpha \mathrm{X}=\mathrm{pXBR}-1$ (from C. Disteche, Department of Pathology, University of Washington, Seattle, Washington); and $\alpha \mathrm{Y}=$ pDP97 (from D. Page, ATCC). (* indicates probes constantly hybridizing to more than one chromosome pair.)

\section{Probe Labeling and Hybridization}

All probes (plasmids) were prepared by alkaline lysis and were labeled by regular nick translation, at a concentration of 10 to $15 \mathrm{ng} / \mathrm{ul}$ of DNA. When the combination algorithm required the same probe to be labeled with two dyes, separate nick-translation reactions were performed, and the labeled DNA combined in the proportions depicted in Figure 1, Table. Separate labeling reactions of 3 to $4 \mu \mathrm{g}$ of every plasmid DNA were performed and stored. Two to three microliters of each labeling reaction were used in separate hybridization experiments to assess hybridization quality, chromosomes yielding signals, and relative signal strength (visual approximation). In the next step, the labeled probes were pooled as depicted in Figure 1 , Table, using $1 \mu \mathrm{l}$ of each labeling reaction for the strong probes (for example, chromosomes 1; 5; 16; $19 ; 3 ; 14 ; 15 ; 17$; and 18 ) and $2 \mu$ of each labeling reaction for the weaker probes. After hybridization, the strength of the various hybridization signals on the chromosomes was visually assessed, and the amount of each labeled probe was adjusted for identification. Ten consecutive experiments were required before the working combination was found. Larger amounts of labeled probe were then combined into a working pool, to be used for 50 to 100 slides. This labeled DNA pool was precipitated, resuspended in hybridization buffer, and stored at $-20^{\circ} \mathrm{C}$. One aliquot $(10 \mu \mathrm{l})$ was used to hybridize one slide.

All labeled nucleotides used in nick translation were custom synthesized in our laboratory, as previously described (Henegariu et al, 2000). The fluorophore- or hapten-dUTP used were: FITC-dUTP, Cy3-dUTP, Cy5-dUTP, DEAC-dUTP, DIG-dUTP, DNP-dUTP, and BIO-dUTP. Labeled antibodies were either purchased or prepared in our laboratory by standard protein-dye conjugation protocols (Molecular Probes, Eugene, Oregon; Amersham Pharmacia, Arlington Heights, Illinois). DIG was detected with sheep anti-DIG (Accurate Chemical \& Scientific, Westbury, New York) conju- gated with DEAC or Cy5.5; DNP was detected with rat anti-DNP (Accurate Chemical \& Scientific) conjugated with Cy5.5; BIO was detected with avidin-AMCA (Accurate Chemical \& Scientific) or avidin (Vector Laboratories, Burlingame, California) conjugated with Cy7. After nick-translation labeling, DNA from every probe was pipetted into the same vial (common pool), according to the volumes depicted in Figure 1, Table. The DNA was ethanol precipitated, resuspended in hybridization buffer (containing 50\% formamide) denatured, and hybridized. Similar results were obtained using hybridization times from 1 hour to 3 days. After hybridization, slides were rinsed in $50 \%$ formamide in 2x SSC for 10 minutes and $0.2 x$ SSC for 15 minutes at $42^{\circ} \mathrm{C}$, followed by antibody incubation. One microliter of each antibody stock solution $(1 \mathrm{mg} / \mathrm{ml})$ was pipetted in the same vial containing $100 \mu$ l of $4 x$ SSC, vortexed, placed on the slide, and covered with a coverslip. After 5 to 10 minutes of incubation at $37^{\circ} \mathrm{C}$, each slide was rinsed for 10 minutes in wash solution (4x SSC, $0.1 \%$ Tween), stained with DAPI (if necessary), dried, mounted with an antifade mounting media, and analyzed. No particular modifications in the common FISH protocol were necessary, as centromeric probes yielded very strong signals.

\section{Image Capturing and Analysis}

Images were captured using a Sensys cooled CCD camera (Photometrics, Tucson, Arizona) and were pseudocolored using either the PowerGene M-FISH package (PSI, Inc.) or generic software (Adobe Photoshop). In CM-FISH, the role of DAPI counterstaining is to provide the location of the marker chromosome in the metaphase. If DAPI staining is not performed (DAPI and AMCA have similar absorption spectra), it can be simply by exposing the metaphase longer (usually 1.5 to 3 seconds) in the DEAC or DAPI/AMCA channel (pseudo-DAPI image). Tissue autofluorescence is more than sufficient to show the chromosomes and help identify the marker (Fig. 1d). This allows the use of avidin AMCA, without the requirement for subsequent coverslip removal, slide rinsing, and DAPI staining. Because CM-FISH is aimed at identifying a marker chromosome (usually with a morphology quite different from the normal chromosomes) in the metaphase, a specialized software package is not necessary for the analysis itself. Images in each channel can be captured using any available system (digital camera, video CCD camera, or cooled CCD camera) and transferred to the hard drive as grayscale images. The six channels plus the DAPI or pseudo-DAPI image (if available) can be merged as a multichannel image with seven channels, using generic imaging software (Adobe Photoshop). Pseudocoloring of all images is also not necessary. As the position of the marker in the metaphase is known from the pseudo-DAPI image, marker analysis is easily performed by simple visualization of its hybridization signals in all six channels. The signal pattern found is compared with a known chart or table (such as Fig. 1, Table) to identify the origin of the marker. In iCM-FISH, 
aneuploidies are identified by counting the number of FISH signals in every fluorescence channel. Overlapping the gray-scale images is useful for precise identification of the origin of every signal. Any FISH software package or generic image analysis software would be equally useful for this purpose.

\section{References}

Choo KH, Vissel B, Nagy A, Earle E, and Kalitsis P (1991). A survey of the genomic distribution of alpha satellite DNA on all of the human chromosomes, and derivation of a new consensus sequence. Nucleic Acids Res 19:1179-1182.

Durm M, Sorokine-Durm I, Haar FM, Hausmann M, Ludwig H, Voisin P, and Cremer C (1998). Fast-FISH technique for rapid, simultaneous labeling of all human centromeres. Cytometry 31:153-162.

Grady DL, Ratliff RL, Robinson DL, McCanlies EC, Meyne J, and Moyzis RK (1992). Highly conserved repetitive DNA sequences are present at human centromeres. Proc Natl Acad Sci U S A 89:1695-1699.

Haddad BR, Schrock E, Meck J, Cowan J, Young H, Ferguson-Smith MA, du Manoir S, and Ried T (1998). Identification of de novo chromosomal markers and derivatives by spectral karyotyping. Hum Genet 103:619-625.

Heller A, Nietzel A, Rocchi M, Starke H, Fiedler W, Claussen $\mathrm{U}$, and Liehr T (2000). Centromere specific multi-color FISH (cenM-FISH) - a new and rapid method for the identification of marker chromosomes. Eur J Hum Genet 8(Suppl 1):87.

Henegariu O, Bray-Ward P, and Ward DC (2000). Custom fluorescent-nucleotide synthesis as an alternative method for nucleic acid labeling. Nat Biotechnol 18:345-348.

Henegariu O, Heerema NA, Bray-Ward P, and Ward DC (1999). Colour-changing karyotyping: An alternative to MFISH/SKY (letter). Nat Genet 23:263-264.

Henegariu O, Heerema NA, Wright LL, Bray-Ward P, Ward DC, and Vance GH (In press, 2001). Improvements in cytogenetic slide preparation: Controlled chromosome spreading, chemical aging and gradual denaturing. Cytometry.

Huang B, Ning Y, Lamb AN, Sandlin CJ, Jamehdor M, Ried T, and Bartley $J$ (1998). Identification of an unusual marker chromosome by spectral karyotyping. Am J Med Genet 80:368-372.

Huegel A, Coyle L, McNeil R, and Smith A (1995). Evaluation of interphase fluorescence in situ hybridization on direct hematological bone marrow smears. Pathology 27:86-90.

Jorgensen AL (1997). Alphoid repetitive DNA in human chromosomes. Dan Med Bull 44:522-534.

Lee C, Wevrick R, Fisher RB, Ferguson-Smith MA, and Lin CC (1997). Human centromeric DNAs. Hum Genet 100:291304.

Magnani I, Meneveri R, Marozzi A, Ginelli E, Fuhrman-Conti AM, Darfler M, Monard SP, and Sacchi N (1998). Functional marker chromosomes lacking detectable alpha satellite DNA: A tool for centromere study. Chromosome Res 6:69-71.
Maratou K, Siddique Y, Kessling AM, and Davies GE (1999). Novel methodology for the detection of chromosome 21specific alpha-satellite DNA sequences. Genomics 57:429432.

Mitchell AR (1996). The mammalian centromere: Its molecular architecture. Mutat Res 372:153-162.

Nietzel A, Starke H, Heller A, Fiedler W, Senger G, Gebhart E, Rocchi M, Claussen U, and Liehr T (1999). Characterization of small marker chromosomes by centromere specific 24color FISH. Cytogenet Cell Genet 85:40.

Pergament E (2000). The application of fluorescence in-situ hybridization to prenatal diagnosis. Curr Opin Obstet Gynecol 12:73-76.

Ried T, Baldini A, Rand TC, and Ward DC (1992). Simultaneous visualization of seven different DNA probes by in situ hybridization using combinatorial fluorescence and digital imaging microscopy. Proc Natl Acad Sci U S A 89:13881392.

Schrock E, du Manoir S, Veldman T, Schoell B, Wienberg J, Ferguson-Smith MA, Ning Y, Ledbetter DH, Bar-Am I, Soenksen D, Garini Y, and Ried T (1996). Multicolor spectral karyotyping of human chromosomes. Science 273:494-497.

Speicher MR, Gwyn Ballard S, and Ward DC (1996). Karyotyping human chromosomes by combinatorial multi-fluor FISH. Nat Genet 12:368-375.

Sullivan BA, Schwartz S, and Willard HF (1996). Centromeres of human chromosomes. Environ Mol Mutagen 28:182-191.

Tanke HJ, Wiegant J, van Gijlswijk RP, Bezrookove V, Pattenier H, Heetebrij RJ, Talman EG, Raap AK, and Vrolijk J (1999). New strategy for multi-colour fluorescence in situ hybridisation: COBRA: COmbined Binary RAtio labelling. Eur J Hum Genet 7:2-11.

Uhrig S, Schuffenhauer S, Fauth C, Wirtz A, Daumer-Haas C, Apacik C, Cohen M, Muller-Navia J, Cremer T, Murken J, and Speicher MR (1999). Multiplex-FISH for pre- and postnatal diagnostic applications. Am J Hum Genet 65:448-462.

Wandall A, Tranebjaerg L, and Tommerup N (1998). A neocentromere on human chromosome 3 without detectable alpha-satellite DNA forms morphologically normal kinetochores. Chromosoma 107:359-365.

Warburton PE, Greig GM, Haaf T, and Willard HF (1991). PCR amplification of chromosome-specific alpha satellite DNA: Definition of centromeric STS markers and polymorphic analysis. Genomics 11:324-333.

Wevrick R and Willard HF (1989). Long-range organization of tandem arrays of alpha satellite DNA at the centromeres of human chromosomes: High-frequency array-length polymorphism and meiotic stability. Proc Natl Acad Sci U S A 86:9394-9398.

Zhao L, Khan Z, Hayes KJ, and Glassman AB (1998). Interphase fluorescence in situ hybridization analysis: $A$ study using centromeric probes 7,8 , and 12. Ann Clin Lab Sci 28:51-56. 